\title{
NEWS, VIEWS, AND COMMENTS
}

\section{Twin Olympians: The Hard Science and the Lives Behind It / Research Summaries: Twin Study of Cardiometabolic Risk Factors; Postnatal Brain Maturation; Parental Responses to Rearing Triplets; Chimaeric Twins / General Interest: Lone Soccer Twin; Identical Triplet Birth; Life and Work of Twin Geoffrey D. Langlands; The Lone Twin}

\author{
Nancy L. Segal \\ Department of Psychology, California State University, Fullerton, CA, USA
}

\begin{abstract}
A look at scientific studies of sports performance is informative in view of the 2012 Olympic games in London. The athletic life histories of selected twin competitors supplement the scientific data. Reviews of recent twin studies concerning cardiometabolic risk factors, postnatal brain maturation, parental responses to rearing triplets, and the birth of an unusual chimaeric twin pair are presented. General interest items include the separate careers of twin soccer players, an unexpected identical triplet birth, the interesting life of twin Geoffrey D. Langlands, and the Lone Twin Boat Project.
\end{abstract}

\section{Twin Olympians: The Hard Science and the Lives Behind It}

The 2012 London Olympics reminded us that twin studies reveal a great deal about factors affecting athletic skills, at all levels of performance. Highlights from two recent comprehensive reviews of this research are summarized below. The life stories of several monozygotic (MZ) twin Olympic participants are also presented. The outcomes of their participation were unknown at the time of writing.

A 2012 review was prepared by Aldo M. Costa and colleagues from the Department of Sports Sciences at the University of Beira Interior, in Covithã, Portugal (Costa et al., 2012). The first section on aerobic endurance reports results from twin studies of peak oxygen uptake. Unadjusted heritability estimates ranged between $40 \%$ and $68 \%$. In a study of 48 young adult twins, the $68 \%$ heritability dropped to $40 \%$ after weight adjustment, and to $26 \%$ after lifestyle factor adjustment. The most recent study, conducted in 2011 with 304 young adult twins, reported a heritability estimate of $71 \%$ following adjustment for weight. The variable findings may reflect sample size differences and procedures.

The genetics of the cardiovascular system have also interested sports scientists. The structure, mass, and function of heart muscle show hereditary effects, as revealed in an

RECEIVED 19 July 2012; ACCEPTED 19 July 2012. First published online 16 November 2012.

ADDRESS FOR CORRESPONDENCE: Nancy L. Segal, Department of Psychology, California State University, Fullerton, CA 92834. E-mail: nsegal@fullerton.edu 
informative table included in the review. Two twin studies reported heritabilities of 0.69 and 0.59 , respectively, for the left ventricular mass and a third study reported values of 0.82 (end disastolic) and 0.93 (end systolic). Measurement precision and sample composition most likely explained the different findings across studies. It was noted that the high heritabilities reported in the third study (Busjahn et al., 2009) were obtained via cardiac magnetic resonance that is a superior measure to echocardiography for obtaining quantitative images of the heart. Interestingly, lung function, while showing genetic effects, does not appear to affect sport performance. However, repeated short intervals of reduced oxygenation, with or without intense endurance activity, can sustain performance if oxygen levels in the atmosphere are low.

A final section of Costa's review concerned anaerobic power and capacity. These measures are related to age, sex, metabolic capacity, muscle mass, higher muscle fiber recruitment, and greater density of fast fibers. Twin studies have indicated genetic influence on strength measures such as static strength (72\%) and maximum strength in leg extension (46\%). Declines in muscle strength and power are likely among the elderly, but longitudinal twin studies capable of identifying changes in genetic and environmental effects on these measures are unavailable.

Short-duration anaerobic tasks have also shown genetic influence. Measures have included handgrip strength and running speed. Twin study findings from long-term anaerobic activities are few, but genetic effects have been noted for maximal lactate concentration and total power.

A second comprehensive review concerned the genetics of physical activity and inactivity, presented by de Vilhena e Santos et al. (2012) from the University of Porto, in Porto, Portugal. Physical activity refers to body movements produced by skeletal muscles that lead to energy expenditure. Physical inactivity refers to sedentary behavior that may be distinct from physical activity, i.e., it does not represent the other end of the physical activity spectrum. The review covers a range of different studies, but only the twin research findings are highlighted.

Twin study findings in this area date to 1981 and represent the efforts of researchers from 24 countries. Classical MZ-DZ (dizygotic) twin comparisons have revealed genetic influence across most measures, for example, physical activity, total physical activity, sports participation, and leisure time physical activity, with some exceptions. Twin studies that organized participants by gender typically found higher heritabilities for males than for females. Age effects were also noted. Increasing genetic effects on sports participation were noted for twins approaching late adolescence and early adulthood, relative to younger twins. In contrast, a Finnish study that assessed twins in their twenties and thirties showed a decline in genetic effects.
Most twin studies reviewed by de Vilhena e Santos et al. (2012) relied on questionnaires and inventories. Studies using behavioral measures would be of interest in the future.

\section{Twins' Sports Histories}

Listed below are brief biographies of Olympian MZ twins. All pair members share abilities, interests and strong social connections to one another as teammates and practice partners.

(1) Grant and Ross James. The James twins were recruited for the rowing team at the University of Wisconsin when a coach spotted the six-foot, five-inch twins lining up for course registration. They agreed, and went on to win world rowing championships in 2008, 2009, and 2011. At age 24, the James twins competed in London (St. Clair, 2012). The twins are said to be 'fraternal, but look a lot alike'.

Given that their rowing coach could distinguish them only when each twin etched a ' $G$ ' or an 'R' on his back, I seriously question this zygosity diagnosis. Photos of the twins also indicate strongly that they are identical. It is likely that their mother, interviewed for the article, was given the wrong determination when the twins were born or is so sensitive to any subtle differences between them that she believes they are fraternal.

(2) Jonathan and Kevin Borlées. Jonathan Borlées is one of Belgium's fastest runners (Van de Velde, 2012). Together with his identical twin brother, Kevin, and sister Olivia, the three siblings participated in the London Olympics. Interestingly, during a March 2012 interview, the reporter observed that Jonathan seemed more concerned with his twin brother's running performance during a race taking place in the United States than he was with his own upcoming race against the famous runner Usain Bolt. The brothers and sisters credit each other for inspiring them to work as hard as possible in competitions.

(3) Carol and Helen Galashan. Carol and Helen Galashan are synchronized divers from Great Britain (Hart, 2012). The identical twin sisters are from England, but represented Scotland in gymnastics until they retired from the sport in 2006. They turned to diving just two and a half years ago. Their matched physical features may well give them a visual advantage over other competitor, as I have suggested previously (Segal, 2000).

(4) Peter and Pavol Hochschomer. The identical Hochschomer twins competed in the London Olympics, representing Slovakia in canoeing (Douglas, 2012). The twins were favorites, having won the slalom C-2 event during the last three summer games. A fourth victory would have been unprecedented; the twins ultimately did not succeed. 


\section{Research Summaries}

\section{Study of Cardiometabolic Risk Factors}

Cardiometabolic risk factors refer to risks for diabetes and heart disease. According to a recent twin study by Hungarian investigators (Jermendy et al., 2011), people residing in Central European areas are at elevated risk for cardiovascular morbidity and mortality, relative to people residing in other European areas. However, regional differences in diabetes-2 risk have not been observed.

Twins in this study included 101 adult twin pairs (63 $\mathrm{MZ}$ and $38 \mathrm{DZ}$ ), recruited in Hungary via twin meetings and media advertisements. This was the first such study conducted in that country. A wide range of clinical and laboratory measures were obtained from participants, among them smoking, blood pressure, and cholesterol levels. Risk factors that were highly or moderately heritable were weight (88\%), waist circumference (71\%), systolic blood pressure (57.15), diastolic blood pressure $(57.7 \%)$, serum creatine (64.1\%), fibrinogen (59.95), and serum hsCRP (51.9\%; this is a marker of inflammation that correlated with coronary artery disease). A mix of shared and non-shared environmental factors affected other measures, among them fasting blood glucose and fasting insulin. Better understanding of these factors can lead to improved treatment and prevention.

Note that two of this study's authors were MZ twins Ádám and Dávid Tárnoki, who attended the International Twin Congress in Florence, Italy, 2012.

\section{Postnatal Brain Maturation}

A fascinating twin study has linked prenatal growth with postnatal brain maturation in 139 twin pairs $(85 \mathrm{MZ}$ and 54 same-sex DZ), followed from age 5 to 25 years (Raznahan et al., 2012). A matched control group of 172 unrelated non-twins also participated, and were assessed between the ages of 5 and 32 years. Magnetic resonance imaging scans were gathered longitudinally for both groups. Ageappropriate Wechsler IQ data were available for all participants, although the ages of intellectual assessment were not provided.

Many interesting and important findings emerged from this study, among them (1) subtle differences in prenatal growth (based on birth weight, within the normal range of human pregnancies) were linked to brain surface area alterations, and (2) these brain effects were found to impact late-maturing cortices associated with higher cognitive functioning. These results were applicable to both twin groups and the singleton controls.

I noted that the mean IQ scores of the three groups differed, with the MZ twins scoring lowest $(109, S D=$ $12.4)$, followed by the DZ twins $(112, S D=12.2)$ and sin- gletons $(116, S D=12.7)$; see Segal (2012) and reply by Raznahan et al. (2012). The authors did not address these differences, but both twin groups scored significantly below the non-twin group. The authors noted that birth weight does not fully reflect the complexities of prenatal growth, making it likely that prenatal effects beyond those associated with birth weight may explain the twins' lower scores; the unusual prenatal hazards to which twins, especially MZ twins (e.g., transfusion syndrome) may be exposed are well known. Issues concerning prenatal factors and later cognitive functioning are important to address, given that some recent studies have shown that twins do not score below non-twins on general intelligence tests, reversing previous thinking on this issue (Christensen et al., 2006; Posthuma et al., 2000).

\section{Parental Perspectives on Triplets}

Rearing triplets has received less attention than raising twins, presumably because they are less frequent in the population. However, advances in assisted reproductive technologies have increased the number of triplets being born, warranting additional attention to their special circumstances. A qualitative analysis of transcripts provided by seven families raising triplets during the first 4 years has highlighted areas for further study (Jenkins \& Coker, 2010).

Families were identified in a large metropolitan area of the southwestern region of the United States. All parents were interviewed by telephone for between 45 minutes and 2 hours, 45 minutes. Five themes emerged: (1) Confusion over high-risk pregnancy: most families were advised by high-risk medical specialists. However, not all parents fully grasped the meaning and implications of a high-risk pregnancy. (2) Learning to manage three babies: Parents were excited, but apprehensive over caring for three infants. Establishing set procedures and practices was vital in their care. (3) Gathering support and creating community: There was a need to seek assistance from other members of the community. Other families with higher-order multiple birth children proved to be the most helpful. (4) Adjusting relationships and responsibilities: Marital relationships suffered somewhat in that couples rarely had time alone together. Financial issues were problematic in many cases, as were responsibilities toward older children. (5) Opportunities to strengthen triplet families: Participants felt that social workers could have done more toward making parents aware of community resources for triplets. Some services, such as car seat loans and clothing exchanges, are beneficial to all families with multiple birth children, not just triplets.

This study, while informative, drew upon the experiences of just seven families who may not be representative of 
triplet families, in general. Interviews with parents of older triplets might have also been included, limitations that the authors acknowledged.

\section{Chimaeric Twins}

Chorions and amnions are the outer and inner membranes, respectively, surrounding the developing embryo. Approximately one-third of MZ twins have separate chorions, amnions, and placentas, while the other two-thirds have a shared chorion and placenta, but separate amnions. Pairs in a very rare group of $\mathrm{MZ}$ twins share the chorion, amnion, and placenta. In contrast, DZ twins typically have separate chorions, amnions, and placentas (Machin \& Keith, 1999).
However, it is currently recognized that a (presumably) very small number of fraternal twins share a chorion.

The most recent such case occurred in Australia to a couple that spontaneously conceived twin boys, diagnosed as MZ due to their shared chorion (Umstad et al., 2012). However, the twins appeared physically different, causing their parents to question the diagnosis. Further medical study confirmed that the twins were DZ, although the processes by which they developed a shared chorion continue to be debated. Families with twins whose observations strongly conflict with the twin type provided to them are advised to request additional information and testing.

\section{General Interest}

\section{Lone Soccer Twin}

Identical Brazilian twins, Fabio and Rafael de Silva, played soccer together as 5-year-olds in Petrópolis, a city located a one hour drive from Rio de Janeiro (Hughes, 2012). The twins were born in 1990 . When they turned 11 they were invited to the school in Fluminese where they continued to play. When the twins turned 15 they were noticed by Les Kershaw, then manager of the UK's United youth academy. The United team signed them on and they played together, confusing everyone with their matched appearance. The only distinguishing mark was Fabio's wedding ring, but when Rafael got married that feature was eliminated.

The twins will play on separate teams for the first time in the coming season. Fabio, the left back, is leaving for London's Queens Park Ranger team on loan, while Rafael will remain with United. The decision was made by Manchester's United manager Alex Ferguson after the team lost the top spot. The weakening of a top player whom Rafael can replace, as well Fabio's injuries, also contributed to the shift. However, the brothers will not be playing against each other because of loan agreements that prevent this - United still holds Fabio's contract while he is on loan to the other team. However, if Fabio should change affiliations formally, the twins might challenge one another in the future. That would be a game to watch. There was great anticipation in 1997 when it appeared that identical twin football players Tiki and Ronde Barber would be part of opposing teams during a Thanksgiving weekend, but the game did not take place.

\section{Identical Triplet Birth}

Thirty-one-year-old Allison Penn and her 46-year-old husband Tom sought assisted reproductive technology after Allison failed to become pregnant after trying for 4 years (MSNBC.msn.com, 2011). The procedure succeeded and Allison delivered what is believed to be the first identical triplet set conceived following implantation of a single embryo. The male triplets, Logan, Eli, and Collin, weighed 4 pounds, 12 ounces; 4 pounds; and 4 pounds, 11 ounces, respectively. All three infants are healthy with the exception of Logan who has a non-functioning kidney. The family resides in Long Island, New York.

\section{Life and Work of Twin Geoffrey D. Langlands}

Geoffrey D. Langlands, now age 94, was born in Britain (Walsh, 2012). He was orphaned at age 12 and never married. He is also a twin, although he has seen his twin brother only twice since he was stationed in British India in 1947. There he was witness to events culminating in the partitioning of British India into the states of India and Pakistan. He remained in Pakistan, becoming a legend in his own time. He began as a faculty member at Aitchinson College before relocating to Chitral where he founded the Langlands School and College that is widely known for its excellence in education.

Langlands has decided to retire, but has future plans. Among them are the building of an improved student dormitory in Chitral and the writing of his memoir. He also plans to celebrate his 95th birthday with his twin brother. Virtually no information about Langland's twin brother is available - it is unknown if the twins are identical or fraternal — but that will, hopefully, be revealed in his published life story.

\section{Lone Twin Boat Project}

In the summer 2012, a boat built from a wide array of personal effects (e.g., hockey sticks and a miniature train) traveled different ports in the United Kingdom (Barnett, 2012). More than 1,200 people contributed to the project, 
designed to bring people together and to create a sense of community. Stories behind the objects have also been recorded. The project is directed by Gregg Whelan and Gary Winters, from the Lone Twin Performance Company, a group of leading contemporary artists. Any specific connection to twins could not be determined.

\section{References}

Barnett, L. (2012, May 1). Lone twin's ship that hockey sticks built. The Guardian. Accessed on $17^{\text {th }}$ October, 2012. Retrieved from http://www.guardian. co.uk/culture/2012/may/01/lone-twin-boat-project.

Christensen, K., Petersen, I., Herskind, A.-M., \& Bingley, P. (2006). Twin/singleton differences in intelligence? A Danish nation-wide population-based register study of test scores and classroom assessments. British Medical Journal, 333, 1095.

Costa, A. M., Breitenfeld, L., Silva, A. J., Izquierdo, M., \& Marques, M. C. (2012). Genetic inheritance effects on endurance and muscle strength: An update. Sport Medicine, 42, 449-468.

de Vilhena e Santos, D., Katzmaryzk, P. T., Deabra, A. F. T., \& Maia, J. A. R. (2012). Genetics of physical activity and physical inactivity in humans. Behavior Genetics, 42, 559578.

Douglas, S. (2012, June 27). 2012 London Olympics: Canoeing preview. Accessed on $17^{\text {th }}$ October, 2012. Retrieved from http://www.wdtn.com/dpp/sports/summer_games/ 2012-london-olympics-canoeing-preview.

Hart, S. (2009, February 2). Galashan Twins in Sync for London, 2012. Accessed on $17^{\text {th }}$ October, 2012. Retrieved from http://www.telegraph.co.uk/sport/ olympics/diving/4436121/Galashan-twins-in-synchfor-London-2012.html.

Hughes, R. (2012, July 4). Room for only one twin at retooled Manchester United. New York Times. Accessed on $17^{\text {th }}$ October, 2012. Retrieved from http://www.nytimes.com/2012/07/04/sports/soccer/04ihtsoccer04.html?_r=1\&ref=robhughes.

Jermendy, J., HorvEath, T., Littvay, L., Steinbach, R., Jermendy, A., Tárnoki, A.,... Tárnoki, D. (2011). Effect of genetic and environmental influences on cardiometabolic risk factors:
A twin study. Cardiovascular Diabetology, 10, 1-8 (online) doi:10.1186/1475-2840-10-96.

Machin, G. A., \& Keith, L. G. (1999). An atlas of multiple pregnancy: Biology and pathology. New York: Parthenon.

MSNBC.msn.com (2011, June 11). Woman gives birth to identical triplets. Accessed on $17^{\text {th }}$ October, 2012. Retrieved from http://www.msnbc.msn.com/id/23471625/ns/ health-health_care/t/woman-gives-birth-identical-triplets/

Posthuma, D., De Geus, E. J., Bleichrodt, N., \& Boomsma, D. L. (2000). Twin-singleton differences in intelligence? Twin Research, 3, 83-87.

Raznahan, A., Greenstein, D., Lee, N. R., Clasen, L. S., \& Giedd, J. N. (2012). Prenatal growth in humans and postnatal maturation into late adolescence. Proceedings of the National Academy of Science, 109, 11, 366-11, 371.

Segal, N. L. (2000). Entwined lives: Twins and what they tell us about human behavior. New York: Plume.

Segal (2012). Twins, birth weight, cognition and handedness. Comment on Raznahan, A., et al. (2012). Prenatal growth in humans and postnatal maturation into late adolescence. Proceedings of the National Academy of Sciences, September, 2012, Letter: www.pnas.org/cgi/ doi/10.1073/pnas.1213701109; Reply by Raznahan et al: www.pnas.org/cgi/doi/10.1073/pnas.1214891109.

St. Clair, S. (2012, July 7). DeKalb Twins in Sync for Olympic Rowing Team. Accessed on $17^{\text {th }}$ October, 2012. Retrieved July 18, 2012 from http://articles. chicagotribune.com/2012-07-07/sports/ct-spt-0708-

olympics-dekalb-twins-rowing-20120707_1_jamesbrothers-twins-college-rowing. "Two Lithe Crew," Los Angeles Times, p. C6.

Umstad, M. P., Short, R. V., Wilson, M., \& Craig, J. (2012). Chimaeric twins: Why monochorionicity does not guarantee monozygosity. Australian and New Zealand Journal of $\mathrm{Ob}$ stetrics and Gynaecology, 52, 305-307. Doi: 10.1111/j.1479828X.2012.01445.x.

Van de Velde, H. (2012, March 15). London 2012: Borlées Aim to Make 2012 a Family Outing. Accessed on $17^{\text {th }}$ October, 2012. Retrieved from http://www.bbc.co.uk/sport/0/olympics/17387358.

Walsh, D. (2012, June 9). Briton there at Pakistan's birth stays at 94, a Living Textbook. New York Times, p. A1-A2. 